\title{
Toward the Development of Virtual Surgical Tools to Aid Orthopaedic FE Analyses
}

\author{
Srinivas C. Tadepalli, ${ }^{1,2}$ Kiran H. Shivanna, ${ }^{2}$ Vincent A. Magnotta, ${ }^{2,3}$ \\ Nicole A. Kallemeyn, ${ }^{1,2}$ and Nicole M. Grosland ${ }^{1,2,4}$ \\ ${ }^{1}$ Seamans Center for the Engineering Arts and Sciences, Department of Biomedical Engineering, The University of Iowa, \\ Iowa City, IA 52242, USA \\ ${ }^{2}$ Center for Computer Aided Design, The University of Iowa, 116 Engineering Research Facility, 330 S. Madison Street, \\ Iowa City, IA 52242, USA \\ ${ }^{3}$ Department of Radiology, University of Iowa Hospitals and Clinics, 200 Hawkins Drive, Iowa City, IA 52242, USA \\ ${ }^{4}$ Department of Orthopaedics and Rehabilitation, University of Iowa Hospitals and Clinics, 200 Hawkins Drive, \\ Iowa City, IA 52242, USA
}

Correspondence should be addressed to Nicole M. Grosland, nicole-grosland@uiowa.edu

Received 18 May 2009; Revised 8 October 2009; Accepted 28 October 2009

Academic Editor: João Manuel R. S. Tavares

Copyright (C) 2010 Srinivas C. Tadepalli et al. This is an open access article distributed under the Creative Commons Attribution License, which permits unrestricted use, distribution, and reproduction in any medium, provided the original work is properly cited.

Computational models of joint anatomy and function provide a means for biomechanists, physicians, and physical therapists to understand the effects of repetitive motion, acute injury, and degenerative diseases. Finite element models, for example, may be used to predict the outcome of a surgical intervention or to improve the design of prosthetic implants. Countless models have been developed over the years to address a myriad of orthopaedic procedures. Unfortunately, few studies have incorporated patientspecific models. Historically, baseline anatomic models have been used due to the demands associated with model development. Moreover, surgical simulations impose additional modeling challenges. Current meshing practices do not readily accommodate the inclusion of implants. Our goal is to develop a suite of tools (virtual instruments and guides) which enable surgical procedures to be readily simulated and to facilitate the development of all-hexahedral finite element mesh definitions.

\section{Introduction}

Orthopaedic surgeons use both surgical and nonsurgical techniques to treat musculoskeletal trauma, sports injuries, degenerative diseases, infections, tumors, and congenital conditions. Orthopaedic surgical operations are associated with the rearrangement of both hard and soft tissues, oftentimes leading to dramatic changes in structural geometry. The primary objective of a surgical correction is typically the maintenance or restoration of function. Due to the complexity of the anatomy under consideration and the biomechanical behavior of the tissues, the impact of a surgical procedure may not always be predicted on the basis of the surgeon's intuition and experience alone. Computational modeling using individual tomographic data can provide valuable information for surgeons during the planning stage. Specifically, the finite element method provides a means to predict surgical outcome based on factors such as bony cuts (osteotomy), bone fragment/segment repositioning, the addition of instrumentation, and the host tissue response. Countless models have been developed over the years addressing procedures ranging from fusions to total joint replacements [1-14]. Unfortunately, few studies have incorporated patient-specific models. Historically, baseline anatomic models have been used due to the time devoted solely to model development. Moreover, surgical simulations impose an additional level of complexity and accompanying set of challenges. Current meshing practices do not readily accommodate the inclusion of implants. The challenges that accompany traditional modeling techniques are magnified when an implant is to be introduced in the model. Consequently, the time devoted to mesh development increases considerably, and hence such models may often prove impractical. 
In pursuit of making patient-specific modeling a reality, we have made advancements in automating the patientspecific bony geometry definitions from CT and/or MR image datasets [15-17] and toward easing the development of corresponding patient-specific finite element (FE) mesh definitions via a custom-written software package, IA-FEMesh [18]. Our goal is to further advance these efforts by developing tools to simulate a variety of surgical procedures, thereby interactively incorporating implants into such models. One day, such models may aid the surgeon in preoperative planning or in the engineering of implant design, ultimately resulting in an improved clinical outcome. Toward that end, our goal is to develop a suite of tools which enable the user to readily simulate a surgical procedure and mesh the resulting structure with an all-hexahedral mesh. Historically, commercial preprocessors were developed for traditional engineering applications where the structures of interest can readily be broken down into geometric primitives, thus making hexahedral mesh development feasible. To capture the geometric complexity of anatomic structures often necessitates the use of a tetrahedral mesh. Hexahedral elements, however, are often preferred for their superior numerical performance as compared to tetrahedral elements $[19,20]$. A mathematical argument in favor of the hexahedral element is that the volume defined by one element must be represented by at least five tetrahedral elements, which in turn yields a system matrix that is computationally more expensive. In contrast to the favorable numerical quality of hexahedral meshes, mesh generation is a difficult task.

Herein, we present a general framework for computerassisted planning of orthopaedic interventions based on finite element modeling via the reconstruction of patient's anatomy from 3D image datasets. To date we have developed a prototype program and an easy to use workflow that interacts with IA-FEMesh, allowing the user to perform a series of surgical manipulations on a bony surface. This tool supports the same datatypes utilized by IA-FEMesh enabling the resulting surfaces to be imported into IAFEMesh for mesh generation. Herein we demonstrate these surgical capabilities by simulating and meshing a cervical laminoplasty procedure.

\section{Surgical Simulation Techniques}

To enable the development of patient-/subject-specific models, the generation of an anatomic model begins with a collection of CT and MR images. CT images facilitate the delineation of the bony anatomy while also providing patient-specific material properties, while MR images allow soft tissues such as cartilage, ligaments and tendons, as well as muscles to be defined. The process of delineating the anatomic structures can be performed via a variety of techniques including manual, semiautomated, and fully automated techniques. The ability to define geometrically accurate representations of bony structures has previously been studied by DeVries et al. [21]. While defining the phalanx bones of the hand, good agreement was found between manual raters (Jaccard metric $=0.91$ ) and physical laser scans of the same specimens (surface distance $=0.20 \mathrm{~mm}$ ). To facilitate the creation of the anatomic models both semiautomated techniques such as the expectation-maximization algorithms [16] as well as artificial neural networks (ANNs) [17] have been explored. In this paper, the BRAINS2 software was used to manually segment the regions of interest [2225]. This software offers a variety of tools to facilitate the delineation of anatomical structures including thresholding, region growing, and clipping. These functions were used in conjunction with the manual editing tools to delineate the bones of the hand, wrist, and spine as shown in the following examples. For the models described in this paper, cadaveric specimens were imaged on a Siemens Sensation 64 slice computed tomography (CT) scanner [matrix $=512 \times 512$ pixels, field of view $(\mathrm{FOV})=172 \mathrm{~mm}$, kilovolts peak $(\mathrm{kVp})$ $=120$, current $=94 \mathrm{~mA}$, exposure $=105 \mathrm{~mA}]$. The in-plane resolution for the hand and wrist was $0.34 \mathrm{~mm}$ with a slice thickness of $0.40 \mathrm{~mm}$, while the spine was imaged with an inplane resolution of $0.5 \mathrm{~mm}$ and $0.6 \mathrm{~mm}$ slice thickness. Once the regions of interest were manually delineated, a surface was generated from the binary segmentation, smoothed via Laplacian smoothing, and exported in STL format from BRAINS2.

The surgical simulation tools described here operate on the surface definitions of the anatomical structures. The surfaces generated in BRAINS2 are loaded into the surgical simulation software to initiate surgical planning. The user is provided several tools to manipulate the quality of the initial surface. For example, the ability to subdivide [26], decimate [27], and smooth the triangles of a given surface is afforded to the user. Figure 1 illustrates various triangulated surface definitions for a carpal bone of the wrist (i.e., capitate). Figures 1(a) and 1(b) illustrate the original triangulated mesh represented in shaded and wireframe form, respectively. Figure 1(c) shows the same surface having each triangle subdivided into 4 new triangles. Additionally, Figure $1(\mathrm{~d})$ highlights the ability to decimate the surface, thereby decreasing the total number of triangles representing the surface. Care must be taken when decimating a surface so that the fidelity of the surface definition is not lost. In terms of smoothing, the user is able to use both Laplacian [28] and windowed sinc [29] smoothing functions. Future work will allow the user to visualize changes in the surface representation that result from these operations.

2.1. Cutting a Bone via a Planar Cut. An osteotomy, for example, is a surgical operation whereby a bone is cut to shorten, lengthen, or change its alignment. We have developed tools to cut a bone, thereby yielding two distinct bone segments. Moreover, tools have also been introduced to cut away the bony surface in preparation for implant insertion.

2.1.1. Performing an Osteotomy. To cut a bone, and retain the individual bony segments, a box widget has been introduced. The box may be interactively positioned (translated and rotated) with respect to the bone, the size of which is controlled via handles provided along each face normal of the box widget (Figure 2). Consequently, the user has the 


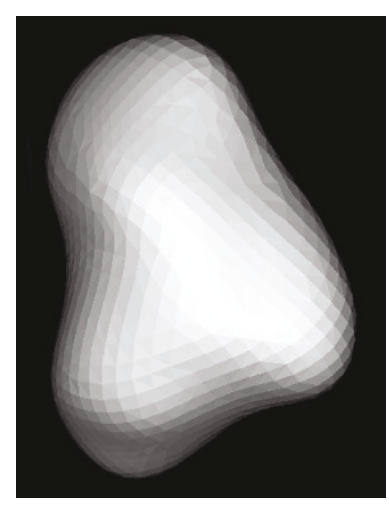

(a)



(b)

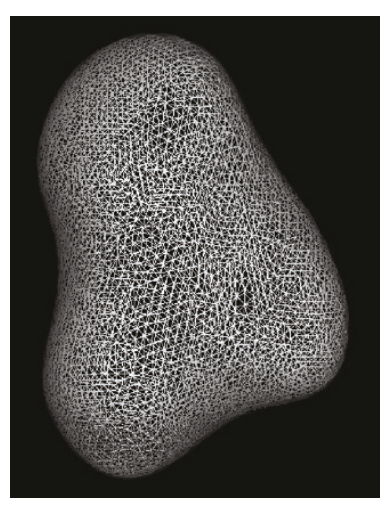

(c)



(d)

Figure 1: The capitate bone-the original triangulated surface represented as (a) a shaded and (b) wireframe surface. (c) Each triangle of the original mesh was subdivided 4 times and $(d)$ the mesh was decimated to reduce the overall number of triangles.

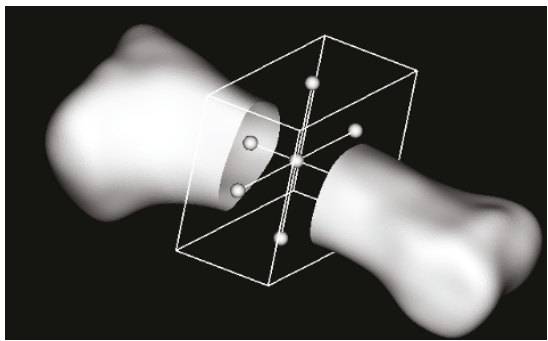

FIGURE 2: Box clip widget used to generate two bony segments.

ability to adjust the box by repositioning each face, thereby enabling a variety of cuts to be simulated. Once the box widget is of the desired length, width, and orientation, the segment of interest (i.e., inside/outside the box) is removed. The surface(s) that results from clipping the original closed surface with the box widget will no longer be closed; consequently, the resulting surfaces must be patched at the location of the simulated cut in order mesh the structure. This has been accomplished using Delaunay triangulation [28]. Rather than maintain a single surface definition, care was taken to assign separate surface definitions to the individual bony segments, thereby permitting the segments to be repositioned relative to one another.

2.1.2. Removing Bone/Bony Surface. To cut a bone in preparation for an implant, planar cuts are often made with the aid of a guide. Consequently, a 3D plane widget available in VTK has been used. The widget is represented by a plane with four corner vertices and a normal vector. Similar to the box widget, the plane can be moved interactively and positioned precisely with respect to the host bone. Thereafter, the desired bony surface is retained and the open face patched.

2.2. Surface Boolean Operations. Boolean operations (intersection, difference, or union) [30] provide the ideal tool for introducing an implant within a host bone (Figure 3 ). The software supports calculations for the intersection and union of two surfaces, as well as the ability to subtract one surface from another. Boolean operations are often used to construct complex objects from simple geometric primitives. We have extended this to include complex anatomic surfaces and surfaces representing implants. The surgical simulation software allows the user to interactively create and size surfaces for simple geometric primitives including cylinders, rectangular blocks, and spheres. In addition, a surface representing a surgical tool and/or implant can be imported and interactively positioned relative to the bone. Once the two surfaces are in the proper position, Boolean operations can be used to manipulate the bony surface. For example, a Boolean operation between a cylindrical surface and the bony surface definition may be used to mimic a drill hole (Figure 4). Again, in order to mesh the structure, the resulting representation must be a closed surface. Consequently, the patching algorithm described previously was used to close the bony surface.

2.3. Meshing the Resulting Surface Definition. Once the surface has been cut/drilled (Figure 4) according to the desired surgical procedure, building blocks may be created and an all-hexahedral FE mesh generated using IA-FEMesh (Figure 5). The meshing algorithms currently available in IAFEMesh dictate that the nodes be projected to the closest point on the surface. Consequently, the position of the building blocks controls the nodal placement. The resulting mesh quality can be evaluated/improved using the tools available in IA-FEMesh [18] prior to exporting the resulting mesh to an FE solver for analysis. Moreover, material properties (user defined and/or image-based) and boundary conditions can be assigned within IA-FEMesh. Although the current meshing practices are feasible as they stand, improvements can be made. For example, during mesh improvement (i.e., smoothing) there is a tendency for the nodes to pull away from the desired surface toward the newly introduced cut/hole (Figure 5). As a result, in the long term we propose to improve upon these meshing strategies by introducing feature edge detection, meshing, and smoothing techniques that preserve these features. 


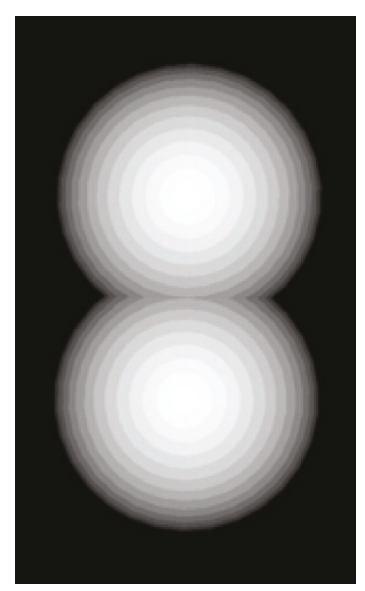

(a)

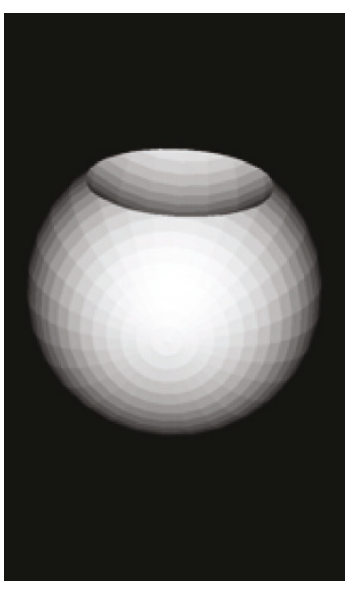

(b)

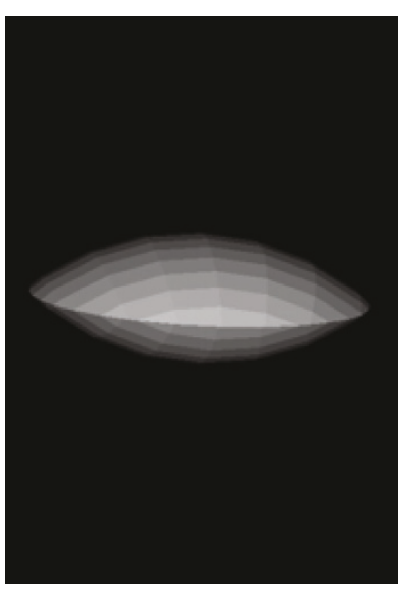

(c)



(d)

Figure 3: Boolean operations performed on (a) two spherical surfaces, (b) subtract, (c) intersection, and (d) union.

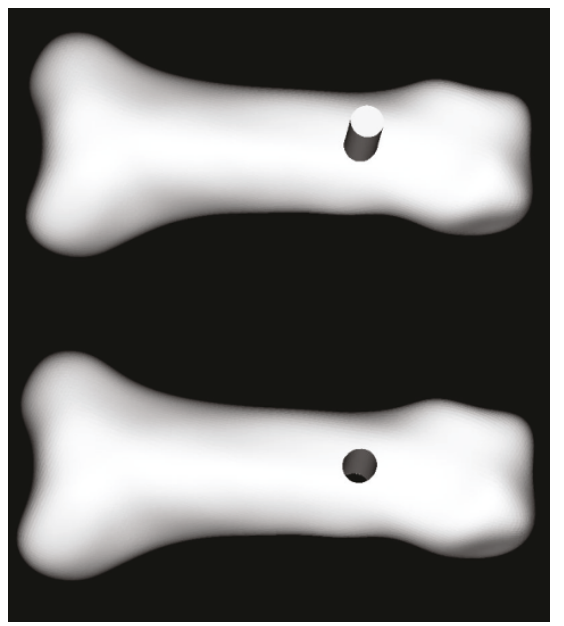

FIgURE 4: Introducing a cylindrical drill hole through the bone.

\section{Results-A Clinically Relevant Application}

While the description above outlines the features of the surgical simulation software, this section describes a clinical application used to test the feasibility of using these tools to evaluate patient-specific surgical procedures.

For example, the procedure of choice for decompression of the cervical spine depends on a variety of factors including the source and location of the compression, the number of vertebral segments involved, cervical alignment, and surgeon experience [31]. Consider, for example, cervical laminoplasty. Laminoplasty was originally developed in Japan [32] to avoid the delayed sequelae of laminectomy without fusion. This procedure initially gained popularity as a treatment for ossification of the posterior longitudinal ligament, but is increasingly being used to treat cases of cervical spondylotic myelopathy. Nevertheless, controversy persists as to whether or not cervical laminoplasty should become the treatment of choice for multilevel cervical stenosis with myelopathy.
Laminoplasty increases the effective diameter of the spinal canal by shifting the laminae dorsally with use of either a single door with a single lateral hinge, or a double door with lateral hinges on both sides. In contrast to laminectomy, laminoplasty retains a covering of the posterior laminar bone and ligamentum flavum over the spinal cord thereby minimizing instability, limits constriction of the dura from extradural scar formation [33, 34], and obviates the need for fusion. Early descriptions of laminoplasty kept the door open with use of suture or wire tethering the spinous process to the hinge side facet joint or capsular tissue [35]. More recent techniques include insertion of an autogenous spinous process graft, allograft bone, or synthetic spacers to keep the door open. Fixation with use of miniplates fixed to the lamina and lateral mass has been reported by multiple authors, without major complications [36-38].

Despite the success of cervical laminoplasty, questions still remain. To address such questions, we recently applied the surgical tools to simulate a cervical laminoplasty using a miniplate at C5 (Figure 6) [39-41]. For this study, a single cadaveric specimen was imaged as described previously. The C5 vertebral body was manually segmented from the CT dataset and the resulting surface loaded into the software to simulate the surgical procedure. The box widget was used to create a bicortical defect on one side, while a Boolean operation between the bony surface definition and a cylindrical surface was used to create a unicortical, or hinge, defect on the contralateral side. A cylindrical surface was also used to create drill holes on either side of the bicortical defect, while the planar widget was used to resect the spinous process. Thereafter, the resulting surface was meshed using a modified building block technique [42] (Figure 6(f)). The final mesh consisted of 29254 elements. To our knowledge, this is the most refined all-hexahedral mesh of a vertebra reported in the literature. Moreover, the quality of the resulting mesh was as good, if not superior to those developed previously using commercial packages. The minimum, average, and maximum element volumes were $0.053,0.419,5.365$, respectively, with a variance of 0.187 . The 


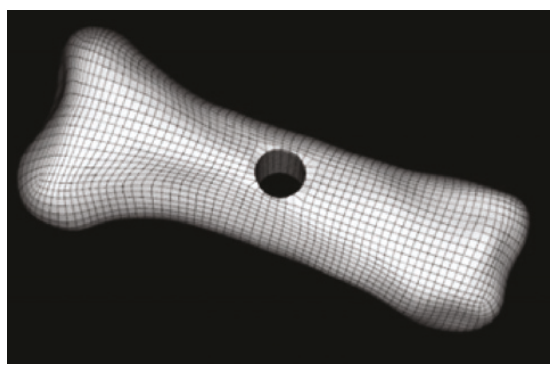

(a)

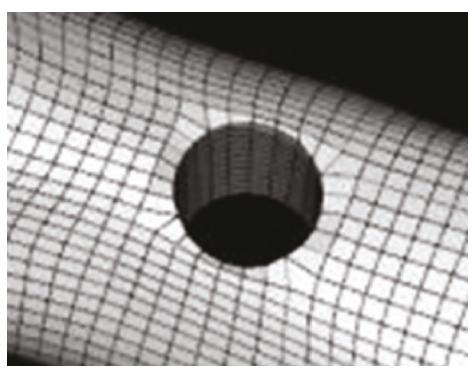

(b)

Figure 5: Multiblock mesh generated about a through hole. The magnified view of the hole illustrates a subtle loss of mesh fidelity due to smoothing immediately adjacent to the hole.

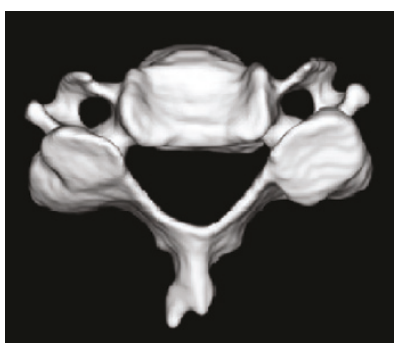

(a)



(e)



(b)

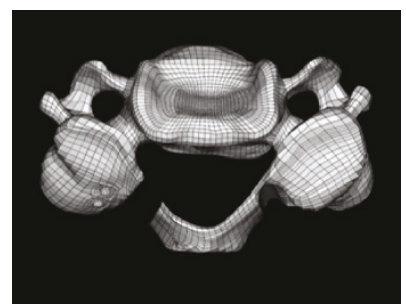

(f)

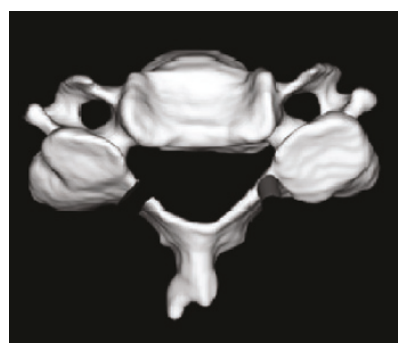

(c)

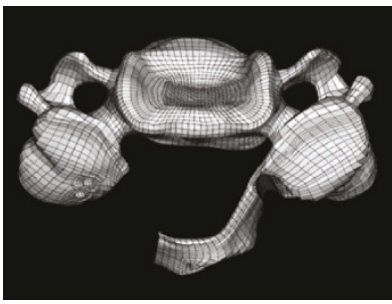

$(\mathrm{g})$

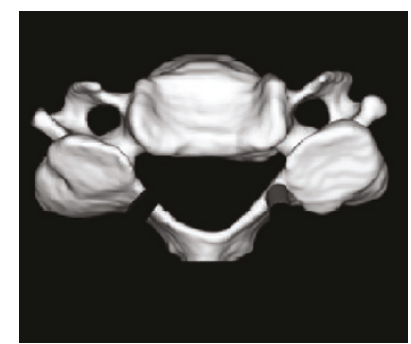

(d)



(h)

Figure 6: Laminoplasty procedure performed on vertebra C5. (a) Intact C5 surface, (b) bicortical defect, (c) contralateral hinge defect introduced, (d) spinous process resected, (e) drill holes introduced, (f) FE mesh, (g) hinge opened and the calculated stresses are input as initial conditions for the (h) plated model.

minimum, average, and maximum Jacobian quality metrics were $0.032,0.339,3.776$, respectively, with a variance of 0.107 . Moreover, FE meshes of the laminoplasty plate and accompanying screws were created. The model was used to predict the potential for fracture at the hinge while opening the posterior elements for plate insertion. Moreover, the stresses induced in the bone as the hinge was opened were incorporated in the plated model as initial conditions. This allowed us to examine the load transfer to the plate/screws as the hinge tried to close postoperatively, in the absence of external loading. The load to failure was predicted by the model under various loading conditions and compared to experimental studies under similar test conditions [39-41].

A substantial increase in the spinal canal area (38\%) and diameter (29\%) was predicted via the FE model, which compared favorably with the measurements obtained experimentally. It was evident from the finite element analysis and cadaveric testing that the introduction of the hinge reduced the strength of the lamina by 5 - to 9 -fold depending on the direction of loading. The stresses in the region of the hinge exceeded the yield strength of the cortical bone indicative of failure, while the stresses in the laminoplasty constructs (i.e., miniplates) were below the yield strength of titanium. Using these meshing techniques, efforts are currently underway to simulate a multilevel laminoplasty in a C27 model and address the flexibility of the spine postoperatively.

\section{Discussion}

The broad objective of our research plan is to augment IAFEMesh with a suite of surgical tools, thereby enabling the software to be used to readily simulate/model a variety of surgical procedures. In pursuit of this objective we have developed an easy to use workflow for the manipulation of surfaces representing anatomical structures to simulate surgical procedures. While some of these features exist in other CAD/CAM software (e.g., SOLIDWORKS, VISI) as 
well as finite element packages (e.g., ABAQUS CAE or ANSYS), implementing a surgical simulation tool within these packages requires a large learning curve, multiple pieces of software with different user interfaces, and significantly more time to generate the model as compared to the workflow presented here. The software presented here provides the same workflow and user interface as utilized in IAFEMesh allowing users to perform the surgical simulations and generate the model in a matter of minutes.

In future work, we will merge the functionality described in this paper directly into the IA-FEMesh software, providing a complete surgical simulation workflow within a single software package. In addition, we are proposing to develop unique technologies to manipulate the anatomic surface definitions, enhance the multiblock meshing practices, and to improve the resulting mesh definitions. A promising means to improve the mesh definition of both anatomic structures and implants has proven to be feature recognition $[43,44]$. This toolkit holds the potential to enable the user to readily simulate surgical interventions, introduce implants, and mesh the resulting models with all-hexahedral elements using multiblock meshing techniques. Our goal is to provide a meshing environment capable of meshing not only anatomic structures, but implants as well. Moreover, establishing the interactions between the two for analysis is imperative. Our long-term goal is to provide a user friendly meshing environment for researchers interested in FE analyses.

Ultimately, these tools and interactions could be coupled with three-dimensional visualization and haptic feedback that could not only serve as a simulation tool, but also a training tool for young physician scientists. This would allow new surgical procedures to be developed and evaluated in mathematical models before transitioning this work to animal models or clinical applications.

\section{Acknowledgments}

The authors gratefully acknowledge the financial support provided in part by an award (R01EB005973) from the National Institute of Biomedical Imaging and Bioengineering, National Institutes of Health and The University of Iowa Presidential Graduate Fellowship.

\section{References}

[1] A. Gebert, J. Peters, N. E. Bishop, F. Westphal, and M. M. Morlock, "Influence of press-fit parameters on the primary stability of uncemented femoral resurfacing implants," Medical Engineering and Physics, vol. 31, no. 1, pp. 160-164, 2009.

[2] Y. S. Lee, T. Q. Lee, and J. H. Keyak, "Effect of an UHMWPE patellar component on stress fields in the patella: a finite element analysis," Knee Surgery, Sports Traumatology, Arthroscopy, vol. 17, no. 1, pp. 71-82, 2009.

[3] T. Zander, A. Rohlmann, and G. Bergmann, "Influence of different artificial disc kinematics on spine biomechanics," Clinical Biomechanics, vol. 24, no. 2, pp. 135-142, 2009.

[4] J. Folgado, P. R. Fernandes, C. R. Jacobs, and V. D. Pellegrini, "Influence of femoral stem geometry, material and extent of porous coating on bone ingrowth and atrophy in cementless total hip arthroplasty: an iterative finite element model," Computer Methods in Biomechanics and Biomedical Engineering, vol. 12, no. 2, pp. 135-145, 2009.

[5] A. Completo, J. A. Simões, and F. Fonseca, "Revision total knee arthroplasty: the influence of femoral stems in load sharing and stability," Knee, vol. 16, no. 4, pp. 275-279, 2009.

[6] M. R. Abdul-Kadir, U. Hansen, R. Klabunde, D. Lucas, and A. Amis, "Finite element modelling of primary hip stem stability: the effect of interference fit," Journal of Biomechanics, vol. 41, no. 3, pp. 587-594, 2008.

[7] Y. H. Kim, O. S. Kwon, and K. Kim, "Analysis of biomechanical effect of stem-end design in revision TKA using digital Korean model," Clinical Biomechanics, vol. 23, no. 7, pp. 853-858, 2008.

[8] S. Checa, M. Taylor, and A. New, "Influence of an interpositional spacer on the behaviour of the tibiofemoral joint: a finite element study," Clinical Biomechanics, vol. 23, no. 8, pp. 1044-1052, 2008.

[9] D. D. D’Lima, N. Steklov, B. J. Fregly, S. A. Banks, and C. W. Colwell Jr., "In vivo contact stresses during activities of daily living after knee arthroplasty," Journal of Orthopaedic Research, vol. 26, no. 12, pp. 1549-1555, 2008.

[10] B. Zielinska and T. L. H. Donahue, "3D finite element model of meniscectomy: changes in joint contact behavior," Journal of Biomechanical Engineering, vol. 128, no. 1, pp. 115-123, 2006.

[11] D. Janssen, J. Stolk, and N. Verdonschot, "Finite element analysis of the long-term fixation strength of cemented ceramic cups," Proceedings of the Institution of Mechanical Engineers, Part H, vol. 220, no. 4, pp. 533-539, 2006.

[12] J. P. Halloran, A. J. Petrella, and P. J. Rullkoetter, "Explicit finite element modeling of total knee replacement mechanics," Journal of Biomechanics, vol. 38, no. 2, pp. 323-331, 2005.

[13] E. Peña, B. Calvo, M. A. Martínez, D. Palanca, and M. Doblaré, "Finite element analysis of the effect of meniscal tears and meniscectomies on human knee biomechanics," Clinical Biomechanics, vol. 20, no. 5, pp. 498-507, 2005.

[14] A. R. Hopkins, U. N. Hansen, and A. A. Amis, "Finite element models of total shoulder replacement: application of boundary conditions," Computer Methods in Biomechanics and Biomedical Engineering, vol. 8, no. 1, pp. 39-44, 2005.

[15] E. Gassman, The validation of an automated image segmentation technique for the development of anatomic finite element models, M.S. thesis, Department of Biomedical Engineering, The University of Iowa, Iowa City, Iowa, USA, 2006.

[16] A. J. Ramme, N. Devries, N. A. Kallemyn, V. A. Magnotta, and N. M. Grosland, "Semi-automated phalanx bone segmentation using the expectation maximization algorithm," Journal of Digital Imaging, vol. 22, no. 5, pp. 483-491, 2009.

[17] E. E. Gassman, S. M. Powell, N. A. Kallemeyn, et al., "Automated bony region identification using artificial neural networks: reliability and validation measurements," Skeletal Radiology, vol. 37, no. 4, pp. 313-319, 2008.

[18] N. M. Grosland, K. H. Shivanna, V. A. Magnotta, et al., "IAFEMesh: an open-source, interactive, multiblock approach to anatomic finite element model development," Computer Methods and Programs in Biomedicine, vol. 94, no. 1, pp. 96$107,2009$.

[19] S. Benzley, E. Perry, K. Merkley, B. Clark, and G. Sjaardema, "A comparison of all-hexahedra and all-tetrahedra finite element meshes for elastic and elasto-plastic analysis," in Proceedings 4th International Meshing Roundtable (IMR '95), pp. 179-191, 1995. 
[20] A. O. Cifuentes and A. Kalbag, "A performance study of tetrahedral and hexahedral elements in 3-D finite element structural analysis," Finite Elements in Analysis and Design, vol. 12, no. 3-4, pp. 313-318, 1992.

[21] N. A. DeVries, E. E. Gassman, N. A. Kallemeyn, K. H. Shivanna, V. A. Magnotta, and N. M. Grosland, "Validation of phalanx bone three-dimensional surface segmentation from computed tomography images using laser scanning," Skeletal Radiology, vol. 37, no. 1, pp. 35-42, 2008.

[22] V. A. Magnotta, G. Harris, N. C. Andreasen, D. S. O'Leary, W. T. C. Yuh, and D. Heckel, "Structural MR image processing using the BRAINS2 toolbox," Computerized Medical Imaging and Graphics, vol. 26, no. 4, pp. 251-264, 2002.

[23] V. A. Magnotta, D. Heckel, N. C. Andreasen, et al., "Measurement of brain structures with artificial neural networks: twoand three-dimensional applications," Radiology, vol. 211, no. 3, pp. 781-790, 1999.

[24] N. C. Andreasen, G. Cohen, G. Harris, et al., "Image processing for the study of brain structure and function: problems and programs," Journal of Neuropsychiatry and Clinical Neurosciences, vol. 4, no. 2, pp. 125-133, 1992.

[25] N. C. Andreasen, T. Cizadlo, G. Harris, et al., "Voxel processing techniques for the antemortem study of neuroanatomy and neuropathology using magnetic resonance imaging," Journal of Neuropsychiatry and Clinical Neurosciences, vol. 5, no. 2, pp. 121-130, 1993.

[26] T. Loop and H.-J. Priebe, "Recovery after anesthesia with remifentanil combined with propofol, desflurane, or sevoflurane for otorhinolaryngeal surgery," Anesthesia and Analgesia, vol. 91, no. 1, pp. 123-129, 2000.

[27] M. Garland and P. S. Heckbert, "Surface simplification using quadric error metrics," in Proceedings of the 24th Annual Conference on Computer Graphics (SIGGRAPH '97), pp. 209216, 1997.

[28] D. A. Field, "Laplacian smoothing and Delaunay triangulations," Communications in Applied Numerical Methods, vol. 4, no. 6, pp. 709-712, 1988.

[29] G. Taubin, T. Zhang, and G. Golub, "Optimal surface smoothing as filter design," in Proceedings of the 4th European Conference on Computer Vision (ECCV '96), pp. 283-292, 1996.

[30] http://staff. science. uva. $\mathrm{nl} / \sim \mathrm{d}$ shamoni/myprojects/Boolean .html.

[31] J. J. Hale, K. I. Gruson, and J. M. Spivak, "Laminoplasty: a review of its role in compressive cervical myelopathy," Spine Journal, vol. 6, supplement 6, pp. S289-S298, 2006.

[32] M. Oyama, S. Hattori, and N. Moriwaki, "A new method of cervical laminoplasty," Central Japan Journal of Orthopaedic Surgery \& Traumatology, vol. 16, pp. 792-794, 1973.

[33] Y. Mikawa, J. Shikata, and T. Yamamuro, "Spinal deformity and instability after multilevel cervical laminectomy," Spine, vol. 12, no. 1, pp. 6-11, 1987.

[34] Y. Ishida, K. Suzuki, K. Ohmori, Y. Kikata, and Y. Hattori, "Critical analysis of extensive cervical laminectomy," Neurosurgery, vol. 24, no. 2, pp. 215-222, 1989.

[35] K. Hirabayashi and K. Satomi, "Operative procedure and results of expansive open-door laminoplasty," Spine, vol. 13, no. 7, pp. 870-876, 1988.

[36] H. Deutsch, P. V. Mummaneni, G. E. Rodts, and R. W. Haid, "Posterior cervical laminoplasty using a new plating system: technical note," Journal of Spinal Disorders and Techniques, vol. 17, no. 4, pp. 317-320, 2004.
[37] M. F. O’Brien, D. Peterson, A. T. H. Casey, and H. A. Crockard, "A novel technique for laminoplasty augmentation of spinal canal area using titanium miniplate stabilization: a computerized morphometric analysis," Spine, vol. 21, no. 4, pp. 474-484, 1996.

[38] T. Goto, K. Ohata, T. Takami, et al., "Hydroxyapatite laminar spacers and titanium miniplates in cervical laminoplasty," Journal of Neurosurgery, vol. 97, supplement 3, pp. 323-329, 2002.

[39] S. Tadepalli, A. Gandhi, D. Fredericks, J. Smucker, and N. Grosland, "Cervical laminoplasty construct stability: experimental and finite element investigation," in Proceedings of the Annual meeting for the American Society of Biomechanics, State College, Pa, USA, August 2009.

[40] S. Tadepalli, N. Kallameyn, K. Shivanna, J. Smucker, D. Fredericks, and N. Grosland, "Cervical laminoplasty construct stability: a finite element study," in Proceedings of the ASME Summer Bioenginering Conference, June 2008.

[41] S. Tadepalli, "Patient-specific hexahedral mesh generation for orthopaedic surgical planning of cervical laminoplasty," in Biomedical Engineering, The University of Iowa, Iowa City, Iowa, USA, 2009.

[42] N. A. Kallemeyn, S. C. Tadepalli, K. H. Shivanna, and N. M. Grosland, "An interactive multiblock approach to meshing the spine," Computer Methods and Programs in Biomedicine, vol. 95, no. 3, pp. 227-235, 2009.

[43] S. C. Tadepalli, K. H. Shivanna, V. A. Magnotta, and N. M. Grosland, "Patient-specific orthopaedic surgical planning: image datasets to FE models," in Proceedings of the 31st Annual Meeting of the American Society of Biomechanics, Palo Alto, CA, 2007.

[44] K. Shivanna, S. Tadepalli, V. Magnotta, and N. Grosland, "Feature based all hexahedral mesh generation in orthopaedic biomechanics," in Proceedings of the American Society of Biomechanics, August 2009. 